\title{
Toward epidemic thresholds on temporal networks: a review and open questions
}

\author{
Jack Leitch ${ }^{1 *}+\left(\mathbb{D}\right.$, Kathleen A. Alexander ${ }^{1,2+}$ and Srijan Sengupta ${ }^{3+}$
}

${ }^{*}$ Correspondence:
jack.leitch@vt.edu
${ }^{\dagger}$ Jack Leitch, Kathleen A. Alexander
and Srijan Sengupta contributed
equally to this work.
${ }^{1}$ Department of Fish and Wildlife
Conservation, Virginia Tech,
Blacksburg, Virginia, USA
Full list of author information is
available at the end of the article

available at the end of the article

\begin{abstract}
Epidemiological contact network models have emerged as an important tool in understanding and predicting spread of infectious disease, due to their capacity to engage individual heterogeneity that may underlie essential dynamics of a particular host-pathogen system. Just as fundamental are the changes that real-world contact networks undergo over time, both independently of and in response to pathogen spreading. These dynamics play a central role in determining whether a disease will die out or become epidemic within a population, known as the epidemic threshold. In this paper, we provide an overview of methods to predict the epidemic threshold for temporal contact network models, and discuss areas that remain unexplored.
\end{abstract}

Keywords: Temporal networks, Epidemiology, Epidemic threshold

\section{Introduction}

Significant advances have been made in the mathematical modeling of infectious diseases since the seminal work of Kermack and McKendrick (1927). The ability to track, measure, predict, and control the spread of contagion has vital implications for the health and well-being of humans, domestic animals, and wildlife. However, there are still many challenges to the creation of models that are able to capture real-world dynamics in complex systems. In this paper, we aim to provide a survey of the important recent developments in infectious disease modeling, specifically temporal contact networks, and identify areas of future research. In particular, we aim to provide an exposition that can be accessible to network scientists and enhance their interest in this topic. We first present well-known epidemic thresholds for temporal networks under the approach of time-scale separation. Next, we introduce three foundational classes of temporal contact network models, with their associated characterizations of the epidemic threshold. We then provide an overview of factors that may influence the epidemic threshold, reviewing work done to date to incorporate these factors into the foundational models. Finally, we present a number of open questions related to computation of epidemic thresholds on temporal networks with broader connections to epidemiology and network theory.

Modeling of infectious disease has taken many different forms, most notably mean-field compartmental models, first introduced by Kermack and McKendrick (1927). Here, individuals are broken up into compartments where a characteristic is shared, as for example,

(C) The Author(s). 2019 Open Access This article is distributed under the terms of the Creative Commons Attribution 4.0 International License (http://creativecommons.org/licenses/by/4.0/), which permits unrestricted use, distribution, and reproduction in any medium, provided you give appropriate credit to the original author(s) and the source, provide a link to the Creative Commons license, and indicate if changes were made. 
disease state (e.g., susceptible (S), infected (I), recovered (R)), reflecting the infection dynamics of a particular host-pathogen system (Keeling and Rohani 2007). While compartmental models are computationally simple, theoretically tractable, and relatively easy to fit to observational data, they can be limited by their fundamental assumptions. Among these is the assumption of homogeneity in mixing, meaning that every individual has equal probability of interacting with every other individual. In reality, however, interactions between individuals vary widely in both number and intensity, and this heterogeneity can significantly impact disease spread (Meyers et al. 2005; Rocha et al. 2011; Galvani and May 2005; Woolhouse et al. 1997). Approaches that allow the transmission function to change based on geospatial or environmental factors can add substantial flexibility and robustness to compartmental models by modifying the strict assumptions on homogeneity (Levin and Durrett 1996; Graham et al. 2004; McCallum et al. 2001; Paull et al. 2012; Engering et al. 2013; White et al. 1996).

In contrast, agent-based models provide maximum flexibility in modeling heterogeneity of individual behavior and potential disease-transmitting contacts with others through stochastic simulation of individuals and their interactions (Alexander et al. 2012). Simulations of contagion spread through the resulting synthetic population can then be used to predict disease dynamics. For example, in Moore et al. (2009), the authors demonstrated that by using agent-based models, ethno-epidemiological data can be integrated in a study of psychostimulant use, which is difficult to accomplish by using compartmental models. However, these models can be computationally intensive for large populations and require incorporation of extensive and detailed knowledge of behavior. Additionally, the fundamental structure and principles of agent-based models preclude analytical treatment, which can be a barrier to intuitive understanding of their dynamics (for a full review, see (Willem et al. 2017)).

In recent years, epidemiological contact network models have emerged as an alternative that engages fundamental heterogeneity of behavior while offering potential for analytical tractability (Keeling 2005). Notable examples where the use of contact networks have led to improvements in prediction or understanding of infectious diseases include (Bengtsson et al. 2015) and (Kramer et al. 2016). In these models each node represents an individual and each edge connecting a pair of nodes represents contact with potential for pathogen transmission. Each individual is assigned a disease state depending on the particulars of the host-pathogen system under study (e.g., SIR, SIS). We can then imagine a spreading process occurring on the network where contagion moves from infected nodes to noninfected nodes. The network structure also makes computation less expensive than agentbased models since the network as a whole can be modeled, instead of tracking each individual agent.

\section{Temporal networks}

In real epidemiological contact networks, however, infectious disease propagation proceeds with concomitant changes in the underlying network (Bansal et al. 2010). Here, contact patterns may change seasonally, or in response to transient conditions that may prompt bursts of contacts (Enright and Kao 2018; Barabási 2005; Hamede et al. 2009; Bharti et al. 2011; Hill et al. 2016). Furthermore, individuals may change their contacts over time either to avoid infection or in direct response to infection (White et al. 2018; Croft et al. 2011; Hawley et al. 2011; Verelst et al. 2016; Schaller 2011). Indeed, a 
growing body of evidence suggests that pathogens can even modify individual host behavior directly in ways that are beneficial to the pathogen (Berdoy et al. 2000; Goodman and Johnson 2011). The assumption that a contact network does not change significantly over time can result in profound mischaracterization of disease spread across the network (Fefferman and Ng 2007). Temporal networks engage these dynamics, providing an opportunity to couple disease spreading and network evolution.

The distinctive characteristic of temporal contact networks is the ordering of contacts over time. Here, pathogen transmission progresses across a temporal network only through time-respecting paths (Holme and Saramäki 2012), which are sequences of edges that follow one after another in time. A time-respecting path could act as a conduit for disease transmission from the starting node through the ending node, whereas a non-time-respecting path could not and is a dead-end for pathogen transmission. When stochastic transitions take place in the network (e.g., the removal of a node), the set of time-respecting paths get adjusted accordingly (e.g., the removed node cannot be a part of time-respecting paths in the future). Indeed, the proportion of paths that are not time-respecting can be considered a rough measure of the importance of modeling disease propagation using a temporal network rather than a static network (Enright and Kao 2018).

The spread of pathogens through a connected population depends on the frequency and strength of interactions between the members of the population. Real-world contact networks are known to exhibit various temporal dynamics, such as concentrated bursts followed by long periods of inaction (Barabási 2005) and periodic patterns due to seasonality (Enright and Kao 2018). Such changes substantially affect the spread on infectious diseases in a population (Zino et al. 2018). Temporal contact networks, therefore, provide a valuable tool for studying the spread of infectious diseases.

\section{Epidemic thresholds}

Particular interest has been given to the question of whether a pathogen, when introduced into an entirely uninfected population, will die out or lead to an epidemic. The outcome is dictated by the parameters of the system with respect to a condition known as the epidemic threshold. More than just a theoretical component, growing evidence provides that these thresholds exist in real-world host-pathogen systems (Dallas et al. 2018). The epidemic threshold is commonly specified in terms of the basic reproduction number, $R_{0}$, defined as the average number of individuals infected by a typical infected individual in an otherwise uninfected population (Diekmann and Heesterbeek 2000). When $R_{0}>1$, the contagion may invade; when $R_{0}<1$, it dies out. More generally, the epidemic threshold can be expressed in terms of the critical value of one or more model parameters. Above the epidemic threshold, the pathogen invades and infects a finite fraction of the population. Below the epidemic threshold, the prevalence (total number of infected individuals) remains infinitesimally small in the limit of large networks (Pastor-Satorras et al. 2015).

Many interventions are developed and implemented based on estimates of this value, seeking either to raise the threshold for the population (e.g., through targeted vaccination) (Shulgin et al. 1998; Wallinga et al. 2005) or to adjust the value of the critical parameter below the threshold (e.g., by reducing probability of transmission of a respiratory pathogen through use of face masks) (Pourbohloul et al. 2005; Meyers et al. 2005). 
Recent years have seen significant advances in estimation of epidemic thresholds for temporal contact networks. Throughout this paper we characterize epidemic threshold conditions using parameters of each particular epidemiological contact network model. Typically these will be stated in terms of the critical transmissibility $\lambda_{c}=\beta / \mu$ of the pathogen, where $\beta$ is the infection rate per effective contact and $\mu$ is the recovery rate, so that an epidemic is likely to occur when $\lambda>\lambda_{c}$. (See Table 1 for notation definition.)

\section{Time scale separation}

Many papers studying disease spread on temporal networks have relied on time-scale separation techniques, an approach which assumes that the rate of spread of a pathogen across the network and the evolution of the network itself take place on distinct time scales. When the spreading process occurs much more rapidly than changes to the network, known as the quenched regime, the network can be fully characterized by a static adjacency matrix (Wang et al. 2003). In the opposite case, the annealed regime, the network changes are assumed to occur much faster than the disease spreading process (Newman 2002). Annealed networks can then be well represented by an average adjacency matrix $\overline{\mathbf{A}}_{k_{i}, k_{j}}$, expressing the probability that two vertices of degree $k_{i}$ and $k_{j}$ are connected in the original network. We defer to Pastor-Satorras et al. (2015) for a thorough review of the topic, but present a few key findings here for comparison with results discussed in later sections.

Under degree-based mean-field (DBMF) theory, the epidemic threshold for uncorrelated annealed networks can be estimated for both SIS dynamics (Pastor-Satorras and Vespignani 2001) and SIR dynamics (Moreno et al. 2002) by

$$
\lambda_{c}=\frac{\langle k\rangle}{\left\langle k^{2}\right\rangle}
$$

Table 1 Common Symbols

\begin{tabular}{|c|c|}
\hline Symbol & Definition and Description \\
\hline $\mathbf{A}, \mathbf{B}, \ldots$ & matrices (bold upper case) \\
\hline $\mathbf{I}$ & the $n \times n$ identity matrix \\
\hline$\rho[\mathbf{A}]$ & spectral radius (largest eigenvalue) of the matrix $\mathbf{A}$ \\
\hline$\langle\cdot\rangle$ & statistical expectation \\
\hline$k_{i}$ & degree of the node $i$ of the network \\
\hline k & the degree distribution of the network \\
\hline$\langle k\rangle,\left\langle k^{2}\right\rangle$ & first and second moments of the degree distribution of the network \\
\hline$S(t), I(t), R(t)$ & $\begin{array}{l}\text { number of susceptible }(S) \text {, infected }(I) \text {, and recovered/removed }(R) \text { individuals in the } \\
\text { population at time } t\end{array}$ \\
\hline$\beta$ & $\begin{array}{l}\text { infection rate: probability of transmission of a pathogen from an infected individual to a } \\
\text { susceptible individual per effective contact (e.g. contact per unit time in continuous-time } \\
\text { models, or per time step in discrete-time models) }\end{array}$ \\
\hline$\mu$ & $\begin{array}{l}\text { recovery rate: probability that an infected individual will recover per unit time (in } \\
\text { continuous-time models) or per time step (in discrete-time models) }\end{array}$ \\
\hline$\lambda$ & transmissibility: the infection rate rescaled by $\mu^{-1}$ so that $\lambda=\beta / \mu$ \\
\hline$\lambda_{c}, \beta_{c}$, etc. & critical transmissibility, critical infection rate, etc., that define the epidemic threshold \\
\hline$\omega$ & rewiring rate or mixing rate \\
\hline a & activity rate distribution for activity-driven networks \\
\hline$m$ & $\begin{array}{l}\text { number of connections to other nodes formed by an active node at each time step, for } \\
\text { activity-driven networks }\end{array}$ \\
\hline
\end{tabular}


where $\langle k\rangle$ and $\left\langle k^{2}\right\rangle$ are the first and second moments of the degree distribution of the network, respectively. This expression elegantly relates the critical transmissibility of a pathogen to the moments of the degree distribution, such that it is proportional to the average connectivity of the network and inversely proportional to fluctuations in the connectivity. Because annealed networks are characterized by their average adjacency matrix, the epidemic threshold described in Eq. (1) can be considered exact for annealed networks.

DBMF theory can be improved by accounting for the inability for a node to infect the node that infected it (as that node is assumed to be no longer susceptible). Newman (2002) calculated the epidemic threshold in this case as

$$
\lambda_{c}=\frac{\langle k\rangle}{\left\langle k^{2}\right\rangle-\langle k\rangle} .
$$

For a comprehensive review of DBMF theory on networks with degree correlations, see (Boguñá et al. 2003). Other notable contributions in this area include those of (Eguíluz and Klemm 2002) and (Serrano and Boguña 2006), who derived the epidemic threshold for scale-free networks and clustered networks, respectively. In (Colizza and Vespignani 2007), the authors derived the invasion threshold for heterogeneous metapopulation networks.

A key assumption of DBMF is that all nodes with the same degree are considered to be statistically equivalent. Individual-based mean-field (IBMF) theory, on the other hand, considers the individual probability that each node will be in a particular disease state at time $t$, thus it can only be applied to quenched networks. The IBMF approach is valid under the mean field assumption of independence between nodes' infectious states. In (Gómez et al. 2010), the authors proposed a discrete-time formulation of the problem to resolve a family of models that range from the so-called contact process to the so-called reactive process. Under this theory, the exact epidemic threshold for an arbitrary static network is given by

$$
\lambda_{c}=\frac{1}{\rho[\mathbf{A}]},
$$

where $\rho[\mathbf{A}]$ is the spectral radius of the adjacency matrix A. (Boguná and Pastor-Satorras 2002), (Wang et al. 2003), and (Chakrabarti et al. 2008) derived this epidemic threshold for SIS dynamics. In Van Mieghem et al. (2009), the authors established this epidemic threshold result under the $N$-intertwined Markov chain model. (Prakash et al. 2010a) later established that this estimate of the epidemic threshold holds for any disease propagation model and any static network topology. In Wang et al. (2016) and Wang et al. (2017) the authors generalized this result to a broader class of networks. This result can be seen as consistent with the epidemic thresholds described in Eq. (1) and (2). For example, under SIS dynamics, the annealed network equivalent to a given uncorrelated quenched network will have an average adjacency matrix with leading eigenvalue $\left\langle k^{2}\right\rangle /\langle k\rangle$ (Castellano and Pastor-Satorras 2010).

Accurate modeling of real-world contact networks depends on availability of sufficiently detailed contact data, which can be difficult to obtain (Eames et al. 2015). Quenched and annealed networks may still be useful in these cases, as they may allow capture of critical features of the network without requiring full contact information. For example, data that permits estimation of the fraction of time that contact occurs between 
each pair of individuals can inform a quenched network with edges weighted according to contact time (e.g., (Moslonka-Lefebvre et al. 2012; Stehlé et al. 2011; Corner et al. 2003)). Though this approach neglects the ordering of contacts, it may provide actionable insights.

\section{Foundational temporal network models}

Over time, a few temporal contact network models have become established in the literature as a foundation on which the study of epidemic thresholds on temporal networks has been constructed. Here we introduce these models along with their associated characterizations of the epidemic threshold.

\section{Neighbor-exchange model}

Volz and Meyers (2009) examined the effect of social mixing on SIR disease spreading for a family of temporal networks, providing an important early result that the computational characterization of the epidemic threshold depends not only on disease parameters of the system and static network topology, but also the rate at which the network changes over time. In this model, nodes are assigned a fixed random node degree according to a configuration model (Molloy and Reed 1998). Then neighbor exchanges, in which pairs of edges are selected uniformly randomly and instantly swapped, occur as a Poisson process at a fixed mixing rate, $\omega$. Thus each individual maintains a fixed number of concurrent contacts while the identities of the contacts change stochastically over time.

Considering the probability generating function $g(k)$ for the degree distribution, the critical transmission rate is given by

$$
\beta_{c}=\frac{\mu(\mu+\omega) g^{\prime}(1)}{(\mu+\omega) g^{\prime \prime}(1)+(\omega-\mu) g^{\prime}(1)},
$$

and the critical mixing rate by

$$
\omega_{c}=\frac{\mu(\beta+\mu) g^{\prime}(1)-\beta \mu g^{\prime \prime}(1)}{\beta g^{\prime \prime}(1)+(\beta-\mu) g^{\prime}(1)} .
$$

The relation for the critical mixing rate in Eq. (5) establishes two special conditions for the mixing rate: When $\lambda<\lambda_{\mathrm{lb}}=g^{\prime}(1) /\left(g^{\prime \prime}(1)+g^{\prime}(1)\right)=\langle k\rangle /\left\langle k^{2}\right\rangle$, an epidemic cannot occur even when the mixing rate is arbitrary large. Similarly, when $\lambda>\lambda_{\mathrm{ub}}=$ $g^{\prime}(1) /\left(g^{\prime \prime}(1)-g^{\prime}(1)\right)=\langle k\rangle /\left(\left\langle k^{2}\right\rangle-2\langle k\rangle\right)$, an epidemic may occur even when the mixing rate is zero (defining a static network). Between these two bounds, an epidemic may occur but only when $\omega>\omega_{c}$.

\section{Activity-driven networks}

Perra et al. (2012) introduced the activity-driven network (ADN) model, which permits evolution of the network in discrete time through a flexible Markov process. Each node is assigned a time invariant activity rate $a_{i}$, according to a given probability distribution $a$. At time step $t$, each node becomes active with probability $a_{i} \Delta t$ and forms $m$ connections with $m$ other nodes selected uniformly at random. Disease transmission is then evaluated over all connections, and all connections are cleared prior to the next time step. The epidemic threshold for the activity-driven network with SIS dynamics is

$$
\beta_{c}=\frac{\mu}{m\left(\langle a\rangle+\sqrt{\left\langle a^{2}\right\rangle}\right)} .
$$


Whereas the epidemic threshold in models discussed so far have been specified in terms of the degree distribution, here it computationally arises from the activity rate distribution and the connectivity parameter. This may not be surprising, given that, in a sense, they implicitly define the effective degree distribution of the network.

While not an activity-driven network per se, Taylor et al. (2012) developed a similar model involving random link activation and deletion with per-potential-link $(\alpha)$ and perlink $(\omega)$ rate parameters, respectively. In addition, they introduced a constraint on link activation in terms of the maximum allowable per-node degree $(M)$, finding that this parameter alone could influence the outcome of an epidemic under SIS dynamics for any particular pair of rate parameters $\alpha$ and $\omega$. This suggests the potential for controlling epidemics by simply limiting the total number of contacts per individual. Their model allows for analytic calculation of $R_{0}$, and Taylor et al. were among the first to establish the limited value of $R_{0}$ in predicting epidemic outcome in temporal networks.

Further work has been done on activity-driven networks by (Starnini and PastorSatorras 2013), who examined topological properties of time-aggregated activity-driven networks, and Starnini and Pastor-Satorras (2014), who confirmed the epidemic threshold defined in Eq. (6) from a temporal percolation perspective. Another key contribution was made by Zino et al. (2016) who presented a continuous-time formulation of the problem. In recent work, (Petri and Barrat 2018) and (Iacopini et al. 2019) have extended the activity driven model to higher-order interactions represented by so-called simplicial complexes.

\section{Temporally-switching networks}

Prakash et al. (2010b) examined the epidemic threshold under SIS dynamics for arbitrary temporal networks, represented by a sequence of $T$ static network snapshots with adjacency matrices $\mathcal{A}=\left\{\mathbf{A}_{\mathbf{1}}, \mathbf{A}_{2}, \ldots \mathbf{A}_{\mathbf{T}}\right\}$. They show that the epidemic threshold is then characterized by the condition

$$
\rho[\mathbf{M}]=1, \quad \mathbf{M}=\prod_{t=1}^{T}\left((1-\mu) \mathbf{I}+\beta \mathbf{A}_{\mathbf{t}}\right),
$$

where $\rho[\mathbf{M}]$ is the spectral radius of the system-matrix $\mathbf{M}$. When $T=1$, we recover the epidemic threshold condition (Eq. 3) estimated by Chakrabarti et al. (2008) and others for static networks. Prakash et al. note the generality of their approach, as the period $T$ can be arbitrary, and snapshots may be repeated as desired within the sequence $\mathcal{A}$.

Valdano et al. (2015a) and Valdano et al. (2015b) also derived an estimate of the epidemic threshold for temporally-switching networks, obtaining a similar outcome using a different method. In their model, temporally-aggregated snapshots of arbitrary temporal networks are mapped to a multilayer network defined by directed edges representing potential transmission of a pathogen between individuals from one time step to the next. Reducing the tensor representation of this multilayer network to matrix form and considering the Markov process for transition from one time step to the next under SIS and SIR dynamics, they derive the epidemic threshold condition

$$
\rho[\mathbf{P}]^{1 / T}=1, \quad \mathbf{P}=\prod_{t=1}^{T}\left((1-\mu) \mathbf{I}+\beta \mathbf{A}_{\mathbf{t}}\right),
$$

which is mathematically equivalent to Eq. (7). The matrix $\mathbf{P}$ can be interpreted as encoding in each entry $P_{i j}$ the probability that node $j$ is infected at time $t=T$, and that 
this infection originated at node $i$ at time $t=0$ and traveled along one or more timerespecting paths (which is valid around the disease-free state and under the assumption of non-interaction among paths). Valdano et al. therefore term the matrix $\mathbf{P}$ as infection propagator.

The common approach of Prakash et al. and Valdano et al., of using a sequence of temporally-aggregated network snapshots, appears to be highly flexible, as it imposes no assumptions on the structure of the temporal network, and the estimated epidemic threshold fully incorporates the topological and temporal dynamics of the network. However, the approach of Valdano et al. assumes a period boundary condition, $\mathbf{A}_{(\mathbf{T}+\mathbf{t})} \equiv \mathbf{A}_{\mathbf{t}}$, where the temporal network snapshots are repeated over time. The authors note that this may result in time-respecting paths that are not present in the original temporal network, but whether this affects the estimated epidemic threshold is an open question. In Valdano et al. (2015b), the authors present numerical results indicating that it is possible to determine an optimal minimum period length that will allow for full characterization of the epidemic threshold.

Valdano et al. (2018) later extended their approach to continuous-time temporal networks and found that in weakly-commuting networks (where the adjacency matrix at time $t$ commutes with the aggregated adjacency matrix up to that time), the epidemic threshold is the same as if it were computed on the time-averaged adjacency matrix $\overline{\mathbf{A}}$, as in Eq. (3). Speidel et al. (2016) studied the epidemic threshold of the SIS model on arbitrary temporal networks and established its connection to the commutator norm. Speidel et al. (2017) assert that the epidemic threshold decreases as the time step between network snapshots increases, and is bounded above by the epidemic threshold for the continuous-time model. They also examine commutation (Speidel et al. 2016) of the adjacency matrices, finding that, when all pairs of snapshot adjacency matrices commute, the epidemic threshold is the same as that of a continuous-time temporal network.

\section{Exploring key factors}

Various authors have presented extensions to the base temporal contact network models outlined above, accounting for factors that are believed to affect epidemic dynamics. Broadly, these include characteristics and dynamics of the underlying contact networks, infectious disease dynamics, and the complex interactions that can arise between them, all potentially influencing the epidemic threshold.

\section{Social structure}

Epidemiological contact networks are naturally driven by contacts between individuals and, whether the population under study is human, domestic animals, or wildlife, social structure largely dictates the nature of these contacts. In wildlife, it is believed that social structure has evolved in part to protect populations from spread of infectious disease (Rozins et al. 2018; Sah et al. 2017). In humans as well, widespread social and cultural norms often serve as a barrier to disease spreading (Schaller 2011; Fincher and Thornhill 2012). On the other hand, it is believed that pathogens have evolved in some cases to exploit social structure in order to further their survival. The effect of social structure on disease spreading has been studied extensively for static contact networks (Salathé and Jones 2010; Huang and Li 2007; Wu and Liu 2008; Stegehuis et al. 2016; Liu and $\mathrm{Hu}$ 2005), and inclusion of social 
structure in temporal contact networks may provide crucial insights into real-world systems.

Nadini et al. (2018) recently explored the effect of explicit static community structure on disease spreading in activity-driven networks. In their model, each node is randomly assigned to a single community at time $t=0$, with community sizes taken from a heavytailed distribution. When a node is activated, rather than forming connections with other nodes selected uniformly at random from the entire population, connections are formed with nodes selected uniformly at random from the same community or social group with probability $\mu$ and from a different community or social group with probability $1-\mu$. In this way, strength of the social structure is parameterized by $\mu$. The relation defining the epidemic threshold for these modular activity-driven networks has no closed-form solution, but some conclusions can be drawn from its form. As $\mu \rightarrow 0$, the modular structure of the network vanishes, and the threshold for both SIS and SIR dynamics is the same as in the activity-driven network without modularity (Perra et al. 2012). As $\mu \rightarrow 1$, however, strong modularity induces a difference between the epidemic threshold for SIR and SIS dynamics. When modularity is strong, the effects appear to be driven by infected individuals having an increased probability of contact with members of their own social group or community. For SIR dynamics, these repeated contacts are increasingly with recovered individuals, in which case contagion spread will be reduced, inhibiting pathogen transmission and movement beyond the social group. For SIS dynamics, the movement of individuals from infected to susceptible status allows sustained transmission and the potential for pathogen endemicity depending on group size and pathogen extinction potential. Pathogen persistence in a social group provides greater probability of extra-group transmission and spread.

Though this model incorporates social structure and changing contacts over time, we must also recognize that social structure itself evolves, and, depending on the time scale on which changes occur relative to disease dynamics, may significantly inhibit or promote disease spreading. For example, with the population growth and recovery of the South American sea lion (Otaria flavescens), significant changes in social composition and spatial distribution of colonies has been observed (Grandi et al. 2008), modifications that will have important influence on pathogen transmission and persistence dynamics. Pathogen infection itself can also influence movement behaviors of individuals feeding back to modify dispersal behavior and social structure across the population, as observed in banded mongoose (Mungos mungo) infected with the novel tuberculosis pathogen Mycobacterium mungi (Fairbanks et al. 2014).

\section{Temporal contact patterns}

Fundamental to temporal contact networks is the concept that each individual's social contact patterns can change over time. Holme and Liljeros (2015) examined the effects of these changes on spreading processes, finding that, for a set of empirical networks, exact times and order of contacts is less predictive of disease outbreaks than the beginning and end of contact and the overall intensity of contact during that period.

Real social networks include both strong ties, contacts that are made repeatedly, frequently, or for long periods, as well as weak ties, which are isolated, sporadic, or of short duration. Incorporating both types of contact patterns can be challenging, as strong ties 
typically require models to be non-Markovian, which tends to result in reduced analytic tractability.

Sun et al. (2015) studied the effects of strong and weak ties on disease spreading using activity-driven network models with memory. In their non-Markovian model, an active node with memory of $n_{i}$ previously-contacted nodes will contact a new node with probability $1 /\left(n_{i}+1\right)$ and a previously-contacted node with probability $n_{i} /\left(n_{i}+1\right)$. Through numerical simulation, they found that for SIR dynamics the memory effect increased the epidemic threshold, while for SIS dynamics the memory effect lowered the epidemic threshold. This result was also reported by Karsai et al. (2015), and is consistent with the effects of community structure on epidemic spreading, as described by Nadini et al. (2018).

Most temporal contact network models assume that contacts occur as a Poisson process, but it has been observed that human behavior tends to result in events occurring in concentrated bursts alternating with long periods of inaction. This phenomenon is known as burstiness (Barabási 2005).

Zino et al. (2018) examined the effect of burstiness in contact patterns on disease spreading in temporal networks. To do this, they altered the activity-driven network model by replacing the standard Poisson activation process for each node with a Hawkes process (Hawkes 1971). Four time invariant parameters were assigned to control to each node's Hawkes process: (i) the jump $J_{i}>0$, which defines the strength of the selfexcitement effect, (ii) the forgetting rate $\gamma_{i}>0$, which defines how quickly excitement is forgotten, (iii) an initial activity rate $a_{i}(0)>0$, and (iv) a background activity rate, $\hat{a}_{i}>0$. It is easily seen that if, $\forall i, J_{i}=0$ and $a_{i}(0)=\hat{a}_{i}$, the result is the standard activity-driven network model with Poisson activation process. In the interest of analytic tractability, Zino et al. assume the same Hawkes process for all nodes, therefore assigning the same jump and forgetting rate to each node. Under these assumptions, when $J<\gamma$, the epidemic threshold is given by

$$
\lambda_{c}=\frac{1-\frac{J}{\gamma}}{\langle\hat{a}\rangle+\sqrt{\left\langle\hat{a}^{2}\right\rangle+\frac{J^{2}}{2 \gamma}\langle\hat{a}\rangle}}
$$

When $J=0$, Equation 9 reduces to the epidemic threshold for the SIS model for standard activity-driven networks (Perra et al. 2012). The above epidemic threshold is less than the epidemic threshold for the standard activity-driven network. Estimates of the epidemic threshold for this model using Monte Carlo numerical simulations find that the epidemic threshold is also reduced under SIR dynamics. The key result here is that models that do not account for burstiness may significantly overestimate the epidemic threshold.

In real-world social interactions, it is possible to observe coordinated bursts of contacts. For example, wildlife may gather at a common water source during a draught, temporarily placing them in close contact. To date, the effects of this phenomenon remain unexplored.

Contacts may also exhibit periodic patterns, e.g., due to seasonal changes in behavior and social interaction (Enright and Kao 2018). The effect this has on disease spreading has not been explicitly examined in temporal networks, though most temporal network models appear to be capable of extension by simply repeating the time period. Indeed, this may be a strength of approaches such as the infection propagator of Valdano et al. (2015b), which assumes periodic boundary conditions. 


\section{Node set changes}

Temporal network models typically assume a closed population, but in most populations demographic changes such as births, deaths, emigration, and immigation are likely to occur on a time scale commensurate with the disease spreading process. Indeed, it has long been known from compartmental models that demographic changes can significantly affect epidemic dynamics (Keeling and Rohani 2007).

Guerra et al. (2012) and Demirel et al. (2017) investigated the interaction between continuous network growth and disease spreading. Network growth occurred through preferential attachment (Barabási and Albert 1999), a process known to result in the creation of scale-free networks. Scale-free topologies are known to have no epidemic threshold in the thermodynamic limit (Pastor-Satorras and Vespignani 2001). A real example of this phenomenon was studied in Rushmore et al. (2014), where it was found that highly central individuals in primate social contact networks also tend to be largerbodied individuals who just happen to encounter more pathogens. However, under SIR dynamics, nodes having high degree as a result of preferential attachment are also more likely to become infected and then removed (assuming the rate of recovery is fast enough), thus raising the epidemic threshold.

\section{Disease dynamics}

While significant attention has been given to how network topology and dynamics affect disease spread, comparatively little progress has been made in understanding the impacts of individual heterogeneity on disease dynamics. Individuals can vary in their relative susceptibility, infectivity, latency, and/or duration of the infectious period in realworld populations. Furthermore, these individual parameters may change over time, as these parameters might be associated with aging, changes in reproductive status, or in response to medical treatment. Coinfection can also introduce additional heterogeneity where one pathogen may induce partial immunity (Dietz 1979) or, alternatively, increased susceptibility to another pathogen infecting the same host.

Darbon et al. (2019) examined the importance of accounting for variation in infection duration, reduction or extension of which could result in fewer or greater secondary infections, respectively. They calculated the epidemic threshold for three real-world networks using the infection propagator approach of Valdano et al. (2015b), concluding that failing to account for this type of heterogeneity could result in significant mis-estimation of the epidemic threshold.

\section{Behavioral response to pathogen spreading: adaptive networks}

It is well known that contact patterns may change over time in response to disease spreading. Humans and animals are known to avoid contact with infected individuals or reduce their interactions overall in response to awareness of disease, a phenomenon known as social distancing or protective sequestration (Reluga 2010). Indeed, quarantine or reducing exposure to a pathogen by reducing contact with others is well established as a public health intervention. Individuals may also have reason to increase their interactions in order to achieve exposure to a pathogen, thus promoting disease spread, sometimes in the interest of preventing future infection (Henry 2005; Lopes et al. 2016; Ezenwa et al. 2016; Cole 2006; Aleman et al. 2009). Individual behavior can also be influenced by the clinical response to pathogen infection. For example, symptoms from infection such as lethargy 
may temporally reduce an individual's contacts with others. Pathogens themselves may increase interactions in infected individuals in ways that promote their spread (Poulin 2010; Lefèvre et al. 2009).

Gross et al. (2006) examined epidemic dynamics on discrete-time adaptive networks under SIS disease dynamics. In their model, at each time step, susceptible nodes disconnect from each adjacent infected node with probability $\omega$ (the re-wiring rate) and form a new link with another susceptible node selected uniformly at random. Thus individuals protectively modify their contact patterns in response to local knowledge of the disease spreading process. This rewiring process introduces spreading dynamics that are not found in static networks, including bistability characterized by two thresholds: the persistence threshold, which is the minimum transmissibility for an already-established disease to remain endemic, and the invasion threshold, which is equivalent to the epidemic threshold that we examine herein (Marceau et al. 2010). Under this adaptive rewiring behavior, Gross et al. find that the epidemic threshold is characterized by

$$
\beta_{c}=\frac{\omega}{\langle k\rangle[1-\exp (-\omega / \mu)]} .
$$

Risau-Gusman and Zanette (2009) consider a model based on Gross et al. (2006), but rewiring at each time step with a node selected uniformly at random from the entire population, irrespective of the target node's disease status. Under this arguably more realistic model, the epidemic threshold is characterized by

$$
\beta_{c}=\frac{\omega+\mu}{\mu\langle k\rangle-\mu} \text {. }
$$

One key dynamic observed by Gross et al. is that, while rewiring acts as a barrier to a disease becoming epidemic, over time it also induces formation of closely-connected communities comprised solely of susceptible individuals. Because they are more densely connected, these communities therefore have a lower average epidemic threshold than the entire network. The model of Risau-Gusman and Zanette does not exhibit this effect, as new connections are not formed preferentially with susceptible individuals, but rather without regard to infection status. We note that (Shaw and Schwartz 2008) made an important early contribution to this area by studying the fluctuating dynamics of the SIRS model on adaptive networks.

Rizzo et al. (2014) investigated the effects of decreased activity rate in infected individuals in activity-driven networks. They define activity rate multipliers $\eta_{S}$ and $\eta_{I}$ for susceptible and infected individuals respectively, where $\eta_{I}<\eta_{S}$, then found the epidemic threshold to be given by

$$
\lambda_{c}=\frac{2}{m\left(\left(\eta_{S}+\eta_{I}\right)\langle x\rangle+\sqrt{\left(\eta_{S}-\eta_{I}\right)^{2}\langle x\rangle^{2}+4 \eta_{S} \eta_{I}\left\langle x^{2}\right\rangle}\right)} .
$$

Thus activity reduction for infected individuals increases the epidemic threshold. When $\eta_{S}=\eta_{I}=\eta$, there is no difference in activity rates between susceptible and infected individuals, and the epidemic threshold matches that found by Perra et al. (2012) (Eq. 6).

Kotnis and Kuri (2013) used activity-driven networks to investigate the effects of social distancing in response to global awareness of infectious disease spreading. Their model considers two base activity rates, $a_{h}$ for healthy individuals and $a_{\text {inf }}$ for infecteds, where $a_{h} \geq a_{\text {inf. }}$ Thus infected individuals are assumed to have a potentially lower activity rate 
as a result of either being aware of their infected state or due to clinical symptoms of infection. Susceptible individuals are assigned an activity rate $a_{\text {sus }}(I)=a_{h} e^{-\delta \cdot I(t)}$, incorporating a risk perception factor $\delta$ that causes activity rates to decrease as the number of infecteds $I(t)$ increases. The resulting epidemic threshold for the SIS model is given by

$$
\lambda_{c}=\frac{1}{m\left(a_{h} e^{-\delta I}+a_{\mathrm{inf}}\right)} .
$$

Rizzo et al. (2014) used a similar activity-driven network model to demonstrate that the epidemic threshold increases when susceptibles reduce their activity rate in response to global awareness of infection.

In reality, individuals may not be informed of the global prevalence of infection within the population, and may only be aware of infecteds within their local neighborhood in the network. Hu et al. (2018) considered this case for activity-driven networks using an SAIS model, including an Alert state to represent individual awareness of risk and therefore preventative behavior, based on the number of infected and alert neighbors. Alert individuals adopt a preventative behavior for $h$ time steps, and then return to their normal behavior. This duration $h$ can be used to represent temporary preventative behavior such as wearing masks $(h=1)$ or a more permanent intervention such as vaccination $(h=\infty)$. The authors find that when the duration of the preventative behavior is short, risk awareness has no effect on the epidemic threshold, but longer durations serve to increase the epidemic threshold.

Moinet et al. (2018) examined the effects of awareness in activity-driven networks with memory. To account for adoption of preventative behavior due to awareness as a result of contact with infected individuals, the probability of infection spreading from an infected individual to a susceptible individual $i$ is specified by $\beta_{i}(t)=\beta \exp \left[-\delta n_{I}(i)_{\Delta T}\right]$, where $\delta$ represents the strength of the awareness and $n_{I}(i)_{\Delta T}$ is the proportion of contacts with infected individuals within the time interval $[t-\Delta T, t]$. They report that the epidemic threshold for both SIS and SIR dynamics is unaffected by awareness in activity-driven networks without memory, but awareness increases the epidemic threshold in activitydriven networks with memory. It should be noted, though, that this effect is only seen in finite networks and does not appear to be present in the limit of large networks.

\section{Multiple modes of transmission}

Some pathogens may have more than one method of transmission. For example, human immunodeficiency virus (HIV) may be transmitted horizontally through sexual contact or blood transfer or vertically during pregnancy or childbirth. These modes of transmission are the result of substantially different types of contact between individuals, with each having a different associated probability of transmission (Patel et al. 2014). Additionally, the temporal concurrency or ordering of these different types of contacts may influence the spread of disease through the population as a whole (Morris et al. 2010).

\section{Open questions}

Both theory and epidemiological applications of temporal networks are active areas of continuing research. Despite the importance of explicitly modeling change in networks over time, temporal networks are still much less popular in disease modeling than static 
methods, as the theory and methodology of temporal networks are actively developing areas of study (Pellis et al. 2015).

For classical epidemiological models and most static contact networks, the basic reproduction number, $R_{0}$, is directly related to the spreading extent and therefore the epidemic threshold. Holme and Masuda (2015) observed that this is not necessarily the case for temporal networks. Examining correlations between $R_{0}$ and the spreading extent on a variety of empirical human contact networks, they found that temporal and topological characteristics of temporal networks had different effects on the relationship between $R_{0}$ and epidemic spreading.

In nearly all cases, static and temporal contact network models are composed of closed and isolated populations, but in our globally connected world human contact networks commonly have far-reaching connections. This may be addressed using a combination of contact network models at different population scales. What dynamics emerge when network changes occur over time at these different scales? And, when we require closed models for analytic or computational tractability, can we account statistically for assumed interactions with individuals external to the network?

As typically defined, the epidemic threshold characterizes the conditions (i.e., the parameters of the epidemic model) under which an epidemic is likely to occur. A related problem of interest is to estimate the probability of an epidemic given a particular set of conditions. This could be of substantial practical importance in scenarios where the pathogen transmission rate and the infection rate are known (or can be estimated within a reasonable margin of error), and the problem of interest is to determine the chances of an epidemic in a given population. The probability of epidemic is not typically estimated in conjunction with the epidemic threshold. Perhaps this could be accomplished by framing the epidemic threshold as a function of the likelihood of epidemic rather than as a singular value. This could provide a more nuanced way to measure cost and benefit of interventions.

\section{Random graph models and community detection}

Most of the existing literature on epidemic thresholds considers the network to be deterministic and fully observed. This approach does not allow for uncertainty quantification or future predictions of interactions. In statistical network analysis, there has been a great deal of emphasis on random graph models that can be used to address this issue. Wellstudied random graph models include stochastic blockmodels and its variants (Holland et al. 1983; Karrer and Newman 2011; Sengupta and Chen 2018), latent space models (Hoff et al. 2002; Handcock et al. 2007), and exponential random graph models (Snijders et al. 2006; Robins et al. 2007), to name a few. Well studied statistical models for time-varying networks include dynamic latent space models (Sewell and Chen 2015b; 2016; 2015a), dynamic stochastic blockmodels (Matias and Miele 2017), and temporal exponential random graph models (TERGM) (Krivitsky and Handcock 2014).

Further, the social structure approach of Nadini et al. (2018) explores the situation where different communities have different recovery rates, under the assumption that the community structure of the network is known. In many real-world situations, this information may not be available. In such cases it would be useful to identify the communities by using community detection techniques like spectral clustering (Rohe et al. 2011; Qin and Rohe 2013; Sengupta and Chen 2015; Lei and Rinaldo 2015) or likelihood modularity 
maximization (Bickel and Chen 2009; Zhao et al. 2012). Connecting the existing results on epidemic thresholds to statistical network models and community detection techniques can greatly broaden the applicability of these results.

Understanding community structure can lead to improved efficacy of targeted immunization strategies. Typical community detection approaches assign individuals to a single community, but in real-world networks for social species individuals are often members of more than one community. Recently (Ghalmane et al. 2019) and (Ghalmane et al. 2019) have addressed this issue for static networks by introducing a centrality measure that accounts for overlapping community structure (see (Cherifi et al. 2019) for a discussion of application to targeted immunization). While potentially challenging due to changing community structure over time, extending this approach to temporal networks could provide a method for further optimizing selective immunization and other targeted interventions.

\section{Local structures and network dynamics}

Local structures, i.e., interaction patterns localized to a small part of the network, can play a significant role in the transmission of a diseases through the network. For example, the presence of unusually dense subgraphs or anomalous cliques (fully connected subgraphs) in a network can accelerate the spread of the disease, while on the other hand, chain-like structures can decelerate the spread of the disease. There has been a rich thread of the network science literature focused on detection of such structures (Alon et al. 1998; Dekel et al. 2014; Feige and Ron 2010; Butucea et al. 2013; Verzelen and Arias-Castro 2015; Arias-Castro et al. 2014; Sengupta 2018; Komolafe et al. 2017; Miller et al. 2015; Miller et al. 2010; Singh et al. 2011). Connecting these detection techniques to epidemic thresholds and understanding the impact of local structures on disease dynamics is an important open question.

Network dynamics is another aspect of network structure that has significant influence over the spread of a disease. In particular, changes in network dynamics can have significant impact on disease transmission. In Sengupta and Woodall (2018), the authors pointed out that network monitoring can refer to two cases, where the network is deterministic with some disease or information being propagated through it, and where the network is itself changes over time and is considered to be a time series. The first problem is called fixed network surveillance and a lot of work has been done particularly on computer networks (Jeske et al. 2018). In this framework, the network itself is fixed over time, and the intensity of information or disease propagation through each edge is considered to be randomly evolving over time. The second problem is called random network surveillance and is a rapidly emerging topic in network science (Priebe et al. 2005; Yu et al. 2018; Woodall et al. 2017; Wilson et al. 2016; Zhao et al. 2018a; Zhao et al. 2018b). In this framework, the network itself changes over time following some time series model. Both problems have important connections with disease transmission and epidemic thresholds, and studying these connections is still an open question.

\section{Connecting theory to practice}

While significant advances have been made in the theory of estimating epidemic thresholds on temporal networks, there has been comparatively little progress in understanding how to apply this theory. One key application is that of risk assessment in case of real- 
world infectious diseases and outbreaks. To date, this has largely been an unexplored area of research. One notable recent contribution in this direction was made by Darbon et al. (2018), where the authors linked the epidemic threshold to the prevalence of bovine brucellosis in Italy.

In most of the current literature on epidemic thresholds, the underlying contact network is assumed to be fully known and observed. In practice, however, this is very unlikely to be the case in general. A fully observed sequence of contact networks might be available for small human groups or animal colonies, but it is not feasible or practical to obtain the detailed sequence of contact networks for, say, all residents of New York City. In absence of complete knowledge on how exactly the network changes over time, the epidemic threshold formulae would not allow prediction. In such circumstances, an alternative is to collect small partial samples from the network, and estimate network features (e.g., average degree, largest eigenvalue) related to epidemic thresholds from such samples. The problem of network sampling has been well-studied in the network science community (Dasgupta et al. 2014; Feige 2006; Gjoka et al. 2010; Goldreich and Ron 2008). Compared to current literature where network features are assumed to be fixed, estimated values from network sampling are going to be random, leading to uncertainty in epidemic threshold computation. Génois et al. (2015) consider some approaches to this problem but note their significant limitations.

On the other hand, in certain epidemiological circumstances (e.g., pathogens with an environmental reservoir or aquatic pathogens at smaller scales) it might not be necessary to obtain the full network dynamics in order to make predictions. Rather, a simpler model based on the important parts of the data might be sufficient. Understanding what kind of data is needed in which epidemiological context is an important direction of future research.

As temporal network theory advances, so too will our ability to analyze spreading processes on these networks. Considerable progress has already been made toward estimating epidemic thresholds on temporal networks, but our toolbox still needs to be developed and refined further. We would encourage epidemiologists, disease ecologists, and network scientists to work on these open problems. Further research toward addressing these unknowns will allow us to capture essential biological and ecological factors, thus moving us toward our goal of improved tracking, prediction, and intervention in the spread of infectious disease.

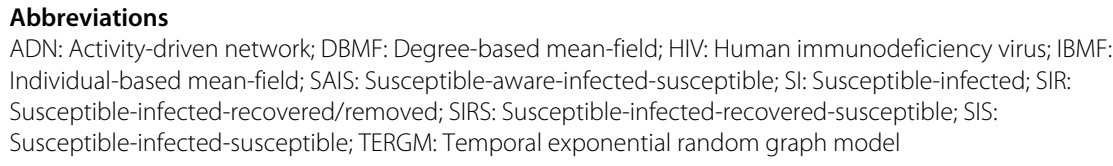

Acknowledgements

Not applicable.

Authors' contributions

$J L$ contributed a review of techniques for estimating epidemic thresholds in static and temporal networks. KA provided connections to modeling concepts in disease ecology and epidemiology. SS provided connections to relevant approaches in network theory. All authors read and approved the final manuscript.

\section{Funding}

Salary support for $J$ was provided by the National Science Foundation (Grant Number 1518663) (KA) as part of the joint NSF-NIH-USDA Ecology and Evolution of Infectious Diseases program. 


\section{Competing interests}

The authors declare that they have no competing interests.

\section{Author details}

${ }^{1}$ Department of Fish and Wildlife Conservation, Virginia Tech, Blacksburg, Virginia, USA. ${ }^{2}$ CARACAL, Centre for Conservation of African Resources: Animals, Communities, and Land Use, Kasane, Botswana. ${ }^{3}$ Department of Statistics Virginia Tech, Blacksburg, Virginia, USA.

\section{Received: 27 March 2019 Accepted: 30 October 2019}

Published online: 14 November 2019

\section{References}

Aleman DM, Wibisono TG, Schwartz B (2009) Accounting for individual behaviors in a pandemic disease spread model. In: Proceedings of the 2009 Winter Simulation Conference (WSC). IEEE. pp 1977-1985. https://doi.org/10.1109/wSC. 2009.5429727

Alexander KA, Lewis BL, Marathe M, Eubank S, Blackburn JK (2012) Modeling of Wildlife-Associated Zoonoses: Applications and Caveats. Vector-Borne Zoonotic Dis 12(12):1005-1018. https://doi.org/10.1089/vbz.2012.0987

Alon N, Krivelevich M, Sudakov B (1998) Finding a large hidden clique in a random graph. Random Struct Algoritm 13(3-4):457-466

Arias-Castro E, Verzelen N, et al. (2014) Community detection in dense random networks. Ann Stat 42(3):940-969

Bansal S, Read J, Pourbohloul B, Meyers LA (2010) The dynamic nature of contact networks in infectious disease epidemiology. J Biol Dyn 4(5):478-489. https://doi.org/10.1080/17513758.2010.503376

Barabási A-L (2005) The origin of bursts and heavy tails in human dynamics. Nature 435(7039):207-211. https://doi.org/ 10.1038/nature03459

Barabási A-L, Albert R (1999) Emergence of Scaling in Random Networks. Science 286(5439):509-512. https://doi.org/10. $1126 /$ science. 286.5439 .509

Bengtsson L, Gaudart J, Lu X, Moore S, Wetter E, Sallah K, Rebaudet S, Piarroux R (2015) Using mobile phone data to predict the spatial spread of cholera. Sci Rep 5:8923

Berdoy M, Webster JP, Macdonald DW (2000) Fatal attraction in rats infected with Toxoplasma gondii. Proc R Soc B Biol Sci 267(1452):1591-1594. https://doi.org/10.1098/rspb.2000.1182

Bharti N, Tatem AJ, Ferrari MJ, Grais RF, Djibo A, Grenfell BT (2011) Explaining Seasonal Fluctuations of Measles in Niger Using Nighttime Lights Imagery. Science 334(6061):1424-1427. https://doi.org/10.1126/science.1210554

Bickel PJ, Chen A (2009) A nonparametric view of network models and Newman-Girvan and other modularities. Proc Nat Acad Sci 106:21068-21073

Boguñá M, Pastor-Satorras R (2002) Epidemic spreading in correlated complex networks. Phys Rev E 66(4):047104. https:// doi.org/10.1103/PhysRevE.66.047104

Boguñá M, Pastor-Satorras R, Vespignani A (2003) Epidemic Spreading in Complex Networks with Degree Correlations(Pastor-Satorras R, Rubi M, Diaz-Guilera A, eds.). Springer, Berlin. https://doi.org/10.1007/978-3-54044943-0_8

Butucea C, Ingster Yl, et al. (2013) Detection of a sparse submatrix of a high-dimensional noisy matrix. Bernoulli 19(5B):2652-2688

Castellano C, Pastor-Satorras R (2010) Thresholds for Epidemic Spreading in Networks. Phys Rev Lett 105(21):218701. https://doi.org/10.1103/PhysRevLett.105.218701

Chakrabarti D, Wang Y, Wang C, Leskovec J, Faloutsos C (2008) Epidemic thresholds in real networks. ACM Trans Inf Syst Secur 10(4):1-26. https://doi.org/10.1145/1284680.1284681

Cherifi H, Palla G, Szymanski BK, Lu X (2019) On community structure in complex networks: challenges and opportunities. 1908.04901

Cole SW (2006) The Complexity of Dynamic Host Networks(Deisboeck TS, Kresh JY, eds.). Springer, Boston. https://doi. org/10.1007/978-0-387-33532-2_26

Colizza V, Vespignani A (2007) Invasion threshold in heterogeneous metapopulation networks. Phys Rev Lett 99:148701. https://doi.org/10.1103/PhysRevLett.99.148701

Corner LAL, Pfeiffer DU, Morris RS (2003) Social-network analysis of Mycobacterium bovis transmission among captive brushtail possums (Trichosurus vulpecula). Prev Vet Med 59(3):147-167. https://doi.org/10.1016/S01675877(03)00075-8

Croft DP, Edenbrow M, Darden SK, Ramnarine IW, van Oosterhout C, Cable J (2011) Effect of gyrodactylid ectoparasites on host behaviour and social network structure in guppies Poecilia reticulata. Behav Ecol Sociobiol 65(12):2219-2227. https://doi.org/10.1007/s00265-011-1230-2

Dallas TA, Krkošek M, Drake JM (2018) Experimental evidence of a pathogen invasion threshold. R Soc Open Sci 5(1):171975. https://doi.org/10.1098/rsos.171975

Darbon A, Valdano E, Poletto C, Giovannini A, Savini L, Candeloro L, Colizza V (2018) Network-based assessment of the vulnerability of italian regions to bovine brucellosis. Prev Vet Med 158:25-34. https://doi.org/10.1016/j.prevetmed. 2018.07.004

Darbon A, Colombi D, Valdano E, Savini L, Giovannini A, Colizza V (2019) Disease persistence on temporal contact networks accounting for heterogeneous infectious periods. R Soc Open Sci 6(1):181404. https://doi.org/10.1098/rsos.181404

Dasgupta A, Kumar R, Sarlos T (2014) On estimating the average degree. In: Proceedings of the 23rd International Conference on World Wide Web, WWW '14. ACM, New York. pp 795-806. https://doi.org/10.1145/2566486.2568019

Dekel Y, Gurel-Gurevich O, Peres Y (2014) Finding hidden cliques in linear time with high probability. Comb Probab Comput 23(1):29-49

Demirel G, Barter E, Gross T (2017) Dynamics of epidemic diseases on a growing adaptive network. Sci Rep 7(1):42352. https://doi.org/10.1038/srep42352 
Diekmann O, Heesterbeek JAP (2000) Mathematical Epidemiology of Infectious Diseases: Model Building, Analysis, and Interpretation. John Wiley \& Sons, Ltd, Chichester

Dietz K (1979) Epidemiologic interference of virus populations. J Math Biol 8(3):291-300. https://doi.org/10.1007/ BF00276314

Eames K, Bansal S, Frost S, Riley S (2015) Six challenges in measuring contact networks for use in modelling. Epidemics 10:72-77. https://doi.org/10.1016/j.epidem.2014.08.006

Eguíluz VM, Klemm K (2002) Epidemic threshold in structured scale-free networks. Phys Rev Lett 89:108701. https://doi. org/10.1103/PhysRevLett.89.108701

Engering A, Hogerwerf L, Slingenbergh J (2013) Pathogen-host-environment interplay and disease emergence. Emerg Microbes Infect 2(1):1-7. https://doi.org/10.1038/emi.2013.5

Enright J, Kao RR (2018) Epidemics on dynamic networks. Epidemics 24:88-97. https://doi.org/10.1016/J.EPIDEM.2018.04. 003

Ezenwa VO, Archie EA, Craft ME, Hawley DM, Martin LB, Moore J, White L (2016) Host behaviour-parasite feedback: an essential link between animal behaviour and disease ecology. Proc R Soc Biol Sci 283(1828):20153078

Fairbanks BM, Hawley DM, Alexander KA (2014) The impact of health status on dispersal behavior in banded mongooses (mungos mungo). EcoHealth 11(2):258-262

Fefferman NH, Ng KL (2007) How disease models in static networks can fail to approximate disease in dynamic networks. Phys Rev E 76(3):031919. https://doi.org/10.1103/PhysRevE.76.031919

Feige U (2006) On sums of independent random variables with unbounded variance and estimating the average degree in a graph. SIAM J Comput 35(4):964-984

Feige U, Ron D (2010) Finding hidden cliques in linear time. In: Discrete Mathematics and Theoretical Computer Science. pp 189-204

Fincher CL, Thornhill R (2012) Parasite-stress promotes in-group assortative sociality: The cases of strong family ties and heightened religiosity. Behav Brain Sci 35(02):61-79. https://doi.org/10.1017/S0140525X11000021

Galvani AP, May RM (2005) Dimensions of superspreading. Nature 438(7066):293-295. https://doi.org/10.1038/438293a

Génois M, Vestergaard CL, Cattuto C, Barrat A (2015) Compensating for population sampling in simulations of epidemic spread on temporal contact networks. Nat Commun 6(1):8860. https://doi.org/10.1038/ncomms9860

Ghalmane Z, El Hassouni M, Cherifi C, Cherifi H (2019) Centrality in modular networks. EPJ Data Sci 8(1):15. https://doi.org/ 10.1140/epjds/s13688-019-0195-7

Gjoka M, Kurant M, Butts CT, Markopoulou A (2010) Walking in facebook: A case study of unbiased sampling of osns. In: 2010 Proceedings IEEE INFOCOM. pp 1-9. https://doi.org/10.1109/INFCOM.2010.5462078

Goldreich O, Ron D (2008) Approximating average parameters of graphs. Random Struct Algoritm 32(4):473-493

Gómez S, Arenas A, Borge-Holthoefer J, Meloni S, Moreno Y (2010) Discrete-time markov chain approach to contact-based disease spreading in complex networks. EPL (Europhys Lett) 89(3):38009

Goodman BA, Johnson PTJ (2011) Disease and the Extended Phenotype: Parasites Control Host Performance and Survival through Induced Changes in Body Plan. PLoS ONE 6(5):20193. https://doi.org/10.1371/journal.pone.0020193

Graham A, Atkinson PM, Danson F (2004) Spatial analysis for epidemiology. Acta Trop 91 (3):219-225

Grandi MF, Dans SL, Crespo EA (2008) Social composition and spatial distribution of colonies in an expanding population of south american sea lions. J Mammal 89(5):1218-1228

Gross T, D'Lima CJD, Blasius B (2006) Epidemic Dynamics on an Adaptive Network. Phys Rev Lett 96(20):208701. https:// doi.org/10.1103/PhysRevLett.96.208701

Guerra B, Gómez-Gardeñes J, Latora V (2012) Adaptive Growing Networks Coevolving with the Spread of Diseases. Int J Bifurcation Chaos 22(07):1250168. https://doi.org/10.1142/S0218127412501684

Hamede RK, Bashford J, McCallum H, Jones M (2009) Contact networks in a wild Tasmanian devil (Sarcophilus harrisii) population: using social network analysis to reveal seasonal variability in social behaviour and its implications for transmission of devil facial tumour disease. Ecol Lett 12(11):1147-1157. https://doi.org/10.1111/j.1461-0248.2009 01370.x

Handcock MS, Raftery AE, Tantrum JM (2007) Model-based clustering for social networks. J R Stat Soc Ser A 170:301-354

Hawkes AG (1971) Spectra of some self-exciting and mutually exciting point processes. Biometrika 58(1):83-90. https:// doi.org/10.1093/biomet/58.1.83

Hawley DM, Etienne RS, Ezenwa VO, Jolles AE (2011) Does Animal Behavior Underlie Covariation Between Hosts' Exposure to Infectious Agents and Susceptibility to Infection? Implications for Disease Dynamics. Integr Comp Biol 51(4):528-539. https://doi.org/10.1093/icb/icr062

Henry S (2005) A pox on my child: Cool!. The Washington Post, Washington

Hill NJ, Ma EJ, Meixell BW, Lindberg MS, Boyce WM, Runstadler JA (2016) Transmission of influenza reflects seasonality of wild birds across the annual cycle. Ecol Lett 19(8):915-925. https://doi.org/10.1111/ele.12629

Hoff PD, Raftery AE, Handcock MS (2002) Latent space approaches to social network analysis. J Am Stat Assoc 97(460):1090-1098

Holland PW, Laskey KB, Leinhardt S (1983) Stochastic blockmodels: first steps. Soc Netw 5:109-137

Holme P, Liljeros F (2015) Birth and death of links control disease spreading in empirical contact networks. Sci Rep 4(1):4999. https://doi.org/10.1038/srep04999

Holme P, Masuda N (2015) The Basic Reproduction Number as a Predictor for Epidemic Outbreaks in Temporal Networks. PLoS ONE 10(3):0120567. https://doi.org/10.1371/journal.pone.0120567

Holme P, Saramäki J (2012) Temporal networks. Phys Rep 519(3):97-125. https://doi.org/10.1016/J.PHYSREP.2012.03.001

Hu P, Ding L, An X (2018) Epidemic spreading with awareness diffusion on activity-driven networks. Phys Rev E 98(6):062322. https://doi.org/10.1103/PhysRevE.98.062322

Huang W, Li C (2007) Epidemic spreading in scale-free networks with community structure. J Stat Mech Theory Exp 2007(01):01014-01014. https://doi.org/10.1088/1742-5468/2007/01/P01014

lacopini I, Petri G, Barrat A, Latora V (2019) Simplicial models of social contagion. Nat Commun 10(1):2485

Jeske DR, Stevens NT, Tartakovsky AG, Wilson JD (2018) Statistical methods for network surveillance. Appl Stoch Model Bus Ind 34(4):425-445 
Karrer B, Newman MEJ (2011) Stochastic blockmodels and community structure in networks. Phys Rev E 83:016107

Karsai M, Perra N, Vespignani A (2015) Time varying networks and the weakness of strong ties. Sci Rep 4(1):4001. https:// doi.org/10.1038/srep04001

Keeling M (2005) The implications of network structure for epidemic dynamics. Theor Popul Biol 67(1):1-8. https://doi. org/10.1016/j.tpb.2004.08.002

Keeling MJ, Rohani P (2007) Modeling Infectious Diseases in Humans and Animals. https://doi.org/10.1097/01.ede. 0000254692.80550 .60

Kermack WO, McKendrick AG (1927) A Contribution to the Mathematical Theory of Epidemics. Proc R Soc A Math Phys Eng Sci 115(772):700-721. https://doi.org/10.1098/rspa.1927.0118

Komolafe T, Quevedo AV, Sengupta S, Woodall WH (2017) Statistical evaluation of spectral methods for anomaly detection in networks. arXiv:1711.01378

Kotnis B, Kuri J (2013) Stochastic analysis of epidemics on adaptive time varying networks. Phys Rev E 87(6):062810. https://doi.org/10.1103/PhysRevE.87.062810

Kramer AM, Pulliam JT, Alexander LW, Park AW, Rohani P, Drake JM (2016) Spatial spread of the west africa ebola epidemic. R Soc Open Sci 3(8):160294. https://doi.org/10.1098/rsos.160294

Krivitsky PN, Handcock MS (2014) A separable model for dynamic networks. J R Stat Soc Ser B (Stat Methodol) 76(1):29-46

Lefèvre T, Adamo SA, Biron DG, Missé D, Hughes D, Thomas F (2009) Invasion of the Body Snatchers: The Diversity and Evolution of Manipulative Strategies in Host-Parasite Interactions. Adv Parasitol 68:45-83. https://doi.org/10.1016/ S0065-308X(08)00603-9

Lei J, Rinaldo A (2015) Consistency of spectral clustering in stochastic block models. Ann Stat 43(1):215-237

Levin SA, Durrett R (1996) From individuals to epidemics. Philos Trans R Soc Lond Ser B Biol Sci 351(1347):1615-1621

Liu Z, Hu B (2005) Epidemic spreading in community networks. Europhys Lett (EPL) 72(2):315-321. https://doi.org/10. 1209/epl/i2004-10550-5

Lopes PC, Block P, König B (2016) Infection-induced behavioural changes reduce connectivity and the potential for disease spread in wild mice contact networks. Sci Rep 6:31790. https://doi.org/10.1038/srep31790

Marceau V, Noël P-A, Hébert-Dufresne L, Allard A, Dubé LJ (2010) Adaptive networks: Coevolution of disease and topology. Phys Rev E 82(3):036116. https://doi.org/10.1103/PhysRevE.82.036116

Matias C, Miele V (2017) Statistical clustering of temporal networks through a dynamic stochastic block model. J R Stat Soc Ser B (Stat Methodol) 79(4):1119-1141

McCallum H, Barlow N, Hone J (2001) How should pathogen transmission be modelled? Trends Ecol Evol 16(6):295-300. https://doi.org/10.1016/S0169-5347(01)02144-9

Meyers LA, Pourbohloul B, Newman MEJ, Skowronski DM, Brunham RC (2005) Network theory and SARS: predicting outbreak diversity. J Theor Biol 232(1):71-81. https://doi.org/10.1016/j.jtbi.2004.07.026

Miller BA, Bliss NT, Wolfe PJ (2010) Toward signal processing theory for graphs and non-Euclidean data. In: Proceedings of the Acoustics Speech and Signal Processing 2010. ICASSP. pp 5414-5417. https://doi.org/10.1109/icassp.2010. 5494930

Miller BA, Beard MS, Wolfe PJ, Bliss NT (2015) A spectral framework for anomalous subgraph detection. IEEE Trans Signal Process 63(16):4191-4206

Moinet A, Pastor-Satorras R, Barrat A (2018) Effect of risk perception on epidemic spreading in temporal networks. Phys Rev E 97(1):012313. https://doi.org/10.1103/PhysRevE.97.012313

Molloy M, Reed B (1998) The Size of the Giant Component of a Random Graph with a Given Degree Sequence. Comb Probab Comput 7(3):295-305

Moore D, Dray A, Green R, Hudson SL, Jenkinson R, Siokou C, Perez P, Bammer G, Maher L, Dietze P (2009) Extending drug ethno-epidemiology using agent-based modelling. Addiction 104(12):1991-1997

Moreno Y, Pastor-Satorras R, Vespignani A (2002) Epidemic outbreaks in complex heterogeneous networks. Eur Phys J B 26(4):521-529. https://doi.org/10.1140/epjb/e20020122

Morris M, Epstein H, Wawer M (2010) Timing Is Everything: International Variations in Historical Sexual Partnership Concurrency and HIV Prevalence. PLOS ONE 5(11):14092. https://doi.org/10.1371/journal.pone.0014092

Moslonka-Lefebvre M, Bonhoeffer S, Alizon S (2012) Weighting for sex acts to understand the spread of STI on networks. J Theor Biol 311:46-53. https://doi.org/10.1016/j.jtbi.2012.06.031

Nadini M, Sun K, Ubaldi E, Starnini M, Rizzo A, Perra N (2018) Epidemic spreading in modular time-varying networks. Sci Rep 8(1):2352. https://doi.org/10.1038/s41598-018-20908-x

Newman MEJ (2002) Spread of epidemic disease on networks. Phys Rev E 66(1):016128. https://doi.org/10.1103/ PhysRevE.66.016128

Pastor-Satorras R, Vespignani A (2001) Epidemic Spreading in Scale-Free Networks. Phys Rev Lett 86(14):3200-3203. https://doi.org/10.1103/PhysRevLett.86.3200

Pastor-Satorras R, Castellano C, Van Mieghem P, Vespignani A (2015) Epidemic processes in complex networks. Rev Mod Phys 87(3):925-979. https://doi.org/10.1103/RevModPhys.87.925

Patel P, Borkowf CB, Brooks JT, Lasry A, Lansky A, Mermin J (2014) Estimating per-act HIV transmission risk. AIDS 28(10):1509-1519. https://doi.org/10.1097/QAD.0000000000000298

Paull SH, Song S, McClure KM, Sackett LC, Kilpatrick AM, Johnson PT (2012) From superspreaders to disease hotspots: linking transmission across hosts and space. Front Ecol Environ 10(2):75-82

Pellis L, Ball F, Bansal S, Eames K, House T, Isham V, Trapman P (2015) Eight challenges for network epidemic models. Epidemics 10:58-62. https://doi.org/10.1016/j.epidem.2014.07.003

Perra N, Gonçalves B, Pastor-Satorras R, Vespignani A (2012) Activity driven modeling of time varying networks. Sci Rep 2(1):469. https://doi.org/10.1038/srep00469

Petri G, Barrat A (2018) Simplicial activity driven model. Phys Rev Lett 121(22):228301

Poulin R (2010) Parasite manipulation of host behavior: an update and frequently asked questions. In: Brockmann $\mathrm{HJ}$ Roper TJ, Naguib M, Wynne-Edwards KE, Mitani JC, Simmons LW (eds). Advances in the Study of Behavior. Academic Press Vol. 41. pp 151-186

Pourbohloul B, Meyers L, Skowronski D, Krajden M, Patrick D, Brunham R (2005) Modeling control strategies of respiratory pathogens. Emerg Infect Dis 11:1249-56. https://doi.org/10.3201/eid1108.040449 
Prakash BA, Chakrabarti D, Faloutsos M, Valler N, Faloutsos C (2010a) Got the Flu (or Mumps)? Check the Eigenvalue! arXiv

Prakash BA, Tong H, Valler N, Faloutsos M, Faloutsos C (2010b) Virus Propagation on Time-Varying Networks: Theory and Immunization Algorithms. In: Balcázar JL, Bonchi F, Gionis A, Sebag M (eds). Machine Learning and Knowledge Discovery in Databases. ECML PKDD 2010. Lecture Notes in Computer Science, Vol 6323. Springer, Berlin. pp 99-114. https://doi.org/10.1007/978-3-642-15939-8_7

Priebe CE, Conroy JM, Marchette DJ, Park Y (2005) Scan statistics on enron graphs. Comput Math Org Theory 11(3):229-247. https://doi.org/10.1007/s10588-005-5378-z

Qin T, Rohe K (2013) Regularized spectral clustering under the degree-corrected stochastic blockmodel. In: Proceedings of the 26th International Conference on Neural Information Processing Systems - Volume 2. Curran Associates Inc., USA. pp 3120-3128

Reluga TC (2010) Game Theory of Social Distancing in Response to an Epidemic. PLoS Comput Biol 6(5):1000793. https:// doi.org/10.1371/journal.pcbi.1000793

Risau-Gusman S, Zanette DH (2009) Contact switching as a control strategy for epidemic outbreaks. J Theor Biol 257(1):52-60. https://doi.org/10.1016/j.jtbi.2008.10.027

Rizzo A, Frasca M, Porfiri M (2014) Effect of individual behavior on epidemic spreading in activity-driven networks. Phys Rev E 90(4):042801. https://doi.org/10.1103/PhysRevE.90.042801

Robins G, Pattison P, Kalish Y, Lusher D (2007) An introduction to exponential random graph ( $p^{*}$ ) models for social networks. Soc Netw 29(2):173-191. https://doi.org/10.1016/j.socnet.2006.08.002. Special Section: Advances in Exponential Random Graph ( $\mathrm{p}^{*}$ ) Models

Rocha LEC, Liljeros F, Holme P (2011) Simulated Epidemics in an Empirical Spatiotemporal Network of 50,185 Sexual Contacts. PLoS Comput Biol 7(3):1001109. https://doi.org/10.1371/journal.pcbi.1001109

Rohe K, Chatterjee S, Yu B (2011) Spectral clustering and the high-dimensional stochastic blockmodel. Ann Stat 39(4):1878-1915

Rozins C, Silk MJ, Croft DP, Delahay RJ, Hodgson DJ, McDonald RA, Weber N, Boots M (2018) Social structure contains epidemics and regulates individual roles in disease transmission in a group-living mammal. Ecol Evol 8(23):12044-12055. https://doi.org/10.1002/ece3.4664

Rushmore J, Caillaud D, Hall RJ, Stumpf RM, Meyers LA, Altizer S (2014) Network-based vaccination improves prospects for disease control in wild chimpanzees. J R Soc Interface 11 (97):20140349

Sah P, Leu ST, Cross PC, Hudson PJ, Bansal S (2017) Unraveling the disease consequences and mechanisms of modular structure in animal social networks. Proc Natl Acad Sci 114(16):4165-4170. https://doi.org/10.1073/pnas.1613616114

Salathé M, Jones JH (2010) Dynamics and Control of Diseases in Networks with Community Structure. PLoS Comput Biol 6(4):1000736. https://doi.org/10.1371/journal.pcbi.1000736

Schaller M (2011) The behavioural immune system and the psychology of human sociality. Philos Trans R Soc B Biol Sci 366(1583):3418-3426. https://doi.org/10.1098/rstb.2011.0029

Sengupta S (2018) Anomaly detection in static networks using egonets. arXiv preprint. arXiv:1807.08925

Sengupta S, Chen Y (2015) Spectral clustering in heterogeneous networks. Stat Sin 25:1081-1106

Sengupta S, Chen Y (2018) A block model for node popularity in networks with community structure. J R Stat Soc Ser B (Stat Methodol) 80(2):365-386

Sengupta S, Woodall WH (2018) Discussion of "Statistical Methods for Network Surveillance" by D. R. Jeske, N.T. Stevens, A. G. Tartakovsky and J. D. Wilson. Appl Stoch Model Bus Ind 34(4):446-448

Serrano MA, Boguñá M (2006) Percolation and epidemic thresholds in clustered networks. Phys Rev Lett 97:088701. https://doi.org/10.1103/PhysRevLett.97.088701

Sewell DK, Chen Y (2015a) Analysis of the formation of the structure of social networks using latent space models for ranked dynamic networks. J R Stat Soc Ser C 64:611-633

Sewell DK, Chen Y (2015b) Latent space models for dynamic networks. J Am Stat Assoc 110(512):1646-1657

Sewell DK, Chen Y (2016) Latent space models for dynamic networks with weighted edges. Soc Netw 44:105-116

Shaw LB, Schwartz IB (2008) Fluctuating epidemics on adaptive networks. Phys Rev E 77(6):066101

Shulgin B, Stone L, Agur Z (1998) Pulse vaccination strategy in the sir epidemic model. Bull Math Biol 60(6):1123-1148. https://doi.org/10.1016/S0092-8240(98)90005-2

Singh N, Miller BA, Bliss NT, Wolfe PJ (2011) Anomalous subgraph detection via sparse principal component analysis. In: 2011 IEEE Statistical Signal Processing Workshop (SSP). pp 485-488. https://doi.org/10.1 109/SSP.2011.5967738

Snijders TAB, Pattison PE, Robins GL, Handcock MS (2006) New specifications for exponential random graph models. Soc Methodol 36(1):99-153. https://doi.org/10.1111/j.1467-9531.2006.00176.x

Speidel L, Klemm K, Eguíluz VM, Masuda N (2016) Temporal interactions facilitate endemicity in the susceptible-infected-susceptible epidemic model. New J Phys 18(7):073013

Speidel L, Klemm K, Eguíluz VM, Masuda N (2017) Epidemic Threshold in Temporally-Switching Networks. In: Masuda N, Holme P (eds). Temporal Network Epidemiology, Chap. 7. Springer, Singapore. pp 161-177. https://doi.org/10.1007/ 978-981-10-5287-3_7

Starnini M, Pastor-Satorras R (2013) Topological properties of a time-integrated activity-driven network. Phys Rev E 87(6):062807. https://doi.org/10.1103/PhysRevE.87.062807

Starnini M, Pastor-Satorras R (2014) Temporal percolation in activity-driven networks. Phys Rev E 89(3):032807. https://doi. org/10.1103/PhysRevE.89.032807

Stegehuis C, van der Hofstad R, van Leeuwaarden JSH (2016) Epidemic spreading on complex networks with community structures. Sci Rep 6(1):29748. https://doi.org/10.1038/srep29748

Stehlé J, Voirin N, Barrat A, Cattuto C, Colizza V, Isella L, Régis C, Pinton J-F, Khanafer N, Van den Broeck W, Vanhems P (2011) Simulation of an SEIR infectious disease model on the dynamic contact network of conference attendees. BMC Med 9(1):87. https://doi.org/10.1186/1741-7015-9-87

Sun K, Baronchelli A, Perra N (2015) Contrasting effects of strong ties on SIR and SIS processes in temporal networks. Eur Phys J B 88(12):326. https://doi.org/10.1140/epjb/e2015-60568-4

Taylor M, Taylor TJ, Kiss IZ (2012) Epidemic threshold and control in a dynamic network. Phys Rev E 85(1):016103. https:// doi.org/10.1103/PhysRevE.85.016103 
Valdano E, Poletto C, Colizza V (2015a) Infection propagator approach to compute epidemic thresholds on temporal networks: impact of immunity and of limited temporal resolution. Eur Phys J B 88(12):341. https://doi.org/10.1140/ epjb/e2015-60620-5

Valdano E, Ferreri L, Poletto C, Colizza V (2015b) Analytical Computation of the Epidemic Threshold on Temporal Networks. Phys Rev X 5(2):021005. https://doi.org/10.1103/PhysRevX.5.021005

Valdano E, Fiorentin MR, Poletto C, Colizza V (2018) Epidemic Threshold in Continuous-Time Evolving Networks. Phys Rev Lett 120(6):068302. https://doi.org/10.1103/PhysRevLett.120.068302

Van Mieghem P, Omic J, Kooij R (2009) Virus spread in networks. IEEE/ACM Trans Netw (TON) 17(1):1-14

Verelst F, Willem L, Beutels P (2016) Behavioural change models for infectious disease transmission: a systematic review (2010-2015). J R Soc Interface 13(125):20160820. https://doi.org/10.1098/rsif.2016.0820

Verzelen N, Arias-Castro E (2015) Community detection in sparse random networks. Ann Appl Probab 25(6):3465-3510. https://doi.org/10.1214/14-AAP1080

Volz E, Meyers LA (2009) Epidemic thresholds in dynamic contact networks. J R Soc Interface 6(32):233-241. https://doi. org/10.1098/rsif.2008.0218

Wallinga J, Heijne JC, Kretzschmar M (2005) A measles epidemic threshold in a highly vaccinated population. PLoS Med 2(11):316

Wang W, Liu Q-H, Zhong L-F, Tang M, Gao H, Stanley HE (2016) Predicting the epidemic threshold of the susceptible-infected-recovered model. Sci Rep 6(1). https://doi.org/10.1038/srep24676

Wang W, Tang M, Stanley HE, Braunstein LA (2017) Unification of theoretical approaches for epidemic spreading on complex networks. Rep Prog Phys 80(3):036603

Wang Y, Chakrabarti D, Wang C, Faloutsos C (2003) Epidemic spreading in real networks: an eigenvalue viewpoint. In: 22nd International Symposium on Reliable Distributed Systems, 2003. Proceedings. IEEE Comput Soc, Florence. pp 25-34. https://doi.org/10.1109/RELDIS.2003.1238052

White A, Begon M, Bowers RG (1996) Host-pathogen systems in a spatially patchy environment. Proc R Soc Lond B Biol Sci 263(1368):325-332

White LA, Forester JD, Craft ME (2018) Covariation between the physiological and behavioral components of pathogen transmission: host heterogeneity determines epidemic outcomes. Oikos 127(4):538-552. https://doi.org/10.1111/oik. 04527

Willem L, Verelst F, Bilcke J, Hens N, Beutels P (2017) Lessons from a decade of individual-based models for infectious disease transmission: A systematic review (2006-2015). BMC Infect Dis 17:612. https://doi.org/10.1186/s12879-0172699-8

Wilson JD, Stevens NT, Woodall WH (2016) Modeling and detecting change in temporal networks via a dynamic degree corrected stochastic block model. arXiv preprint. arXiv:1605.04049

Woodall WH, Zhao MJ, Paynabar K, Sparks R, Wilson JD (2017) An overview and perspective on social network monitoring. IISE Trans 49(3):354-365. https://doi.org/10.1080/0740817X.2016.1213468. http://dx.doi.org/10.1080/0740817X.2016.1213468

Woolhouse MEJ, Dye C, Etard JF, Smith T, Charlwood JD, Garnett GP, Hagan P, Hii JLK, Ndhlovu PD, Quinnell RJ, Watts CH, Chandiwana SK, Anderson RM (1997) Heterogeneities in the transmission of infectious agents: Implications for the design of control programs. Proc Natl Acad Sci 94(1):338-342. https://doi.org/10.1073/pnas.94.1.338

Wu X, Liu Z (2008) How community structure influences epidemic spread in social networks. Phys A Stat Mech Appl 387(2-3):623-630. https://doi.org/10.1016/j.physa.2007.09.039

Yu L, Woodall WH, Tsui K-L (2018) Detecting node propensity changes in the dynamic degree corrected stochastic block model. Soc Netw 54:209-227

Zhao MJ, Driscoll AR, Sengupta S, Stevens NT, Fricker RDJr, Woodall WH (2018a) The effect of temporal aggregation level in social network monitoring. PLOS ONE 13(12):1-21. https://doi.org/10.1371/journal.pone.0209075

Zhao MJ, Driscoll AR, Sengupta S, Fricker Jr RD, Spitzner DJ, Woodall WH (2018b) Performance evaluation of social network anomaly detection using a moving window-based scan method. Qual Reliab Eng Int 34(8):1699-1716

Zhao Y, Levina E, Zhu J (2012) Consistency of community detection in networks under degree-corrected stochastic block models. Ann Stat 40:2266-2292

Zino L, Rizzo A, Porfiri M (2016) Continuous-Time Discrete-Distribution Theory for Activity-Driven Networks. Phys Rev Lett 117(22):228302. https://doi.org/10.1103/PhysRevLett.117.228302

Zino L, Rizzo A, Porfiri M (2018) Modeling Memory Effects in Activity-Driven Networks. SIAM J Appl Dyn Syst 17(4):2830-2854. https://doi.org/10.1137/18M1171485

\section{Publisher's Note}

Springer Nature remains neutral with regard to jurisdictional claims in published maps and institutional affiliations. 